\title{
Antibiogram of Extended Spectrum Beta-lactamase (ESBL) producing Escherichia coli and Klebsiella pneumoniae isolated from Hospital Samples
}

\author{
Iraj Alipourfard, ${ }^{1}$ Nilufar Yeasmin Nili ${ }^{2 *}$ \\ ${ }^{1}$ Shahid Bahonar Hospital of Tehran, Tehran, Iran; ${ }^{2}$ Department of Microbiology, \\ Dhaka Medical College, Dhaka-1000, Bangladesh.
}

\begin{abstract}
Extended spectrum beta-lactmase (ESBL) producing organisms create a major problem for clinical therapeutics. The frequency of ESBL producing strains among clinical isolates has been steadily increasing over the past few years resulting in limitation of the therapeutic options. These resistant bacteria are emerging world wide as a threat to human health in both the community and hospital settings. -lactamase production by several organisms is the most important mechanism of resistance to beta-lactam antibiotics, such as penicillins and cephalosporins. This study was done to determine the susceptibility of different antimicrobials to ESBL producing Escherichia coli and Klebsiella pneumoniae isolated from wound swabs, blood, urine, fluid, tracheal aspirates and sputum in Shahid Bahonar Hospital of Tehran from July, 2007 to June, 2008. A total of 115 ESBLproducing isolates were obtained from outdoor and indoor patients. Out of 115 isolates, $60 \%$ were $E$. coli and 40\% were K. pneumoniae. All ESBL-producing isolates were confirmed using the Clinical and Laboratory Standards Institute (CLSI)-approved double-disk diffusion method. 29.6\% of these isolates were collected from medical wards and $\mathbf{2 4 . 3 \%}$ were collected from outdoor. Urine $\mathbf{( 7 0 . 4 \% )}$ ) was the main source of ESBL-producing isolates from all patients, followed by blood (16.5\%). All isolates were susceptible to both imipenem and meropenem. Of all isolates, $\mathbf{9 3 . 9 \%}$ were susceptible to amikacin. The cephalosporins (1-4 generations) were almost $\mathbf{1 0 0 \%}$ resistant. For Nitrofurantoin, $\mathbf{5 7 . 4 \%}$ were sensitive. High rate resistance $\mathbf{( 7 4 . 8 \% )}$ was observed to all quinolones tested. Aztreonam, Ampicillin, Co-amoxyclav and Ampicillin/Sulbactam were $100 \%$ resistant. This study shows that the frequency of ESBL producing strains of $E$. coli and $K$. pneumoniae is high in both hospital and community levels and it has a significant implication for patients' management. Advance drug resistance surveillance and molecular characteristics of ESBL isolates is necessary to guide the appropriate and judicious antibiotic use.
\end{abstract}

Key words: Extended spectrum beta-lactamase (ESBL), Drug sensitivity, Escherichia coli, Klebseilla pneumoniae.

\section{Introduction}

Extended spectrum beta-lactamase (ESBL) are plasmid mediated, TEM-1, TEM-2 and SHV-1 derived enzymes conferring broad resistance to penicillin, cephalosporin and monobactam but not to carbapenem. ${ }^{1}$ These enzymes are

\section{Correspondence:}

Dr. Nilufar Yeasmin Nili

OSD, DGHS, Mohakhali, Dhaka-1212.

On deputation- Dept. of Microbiology

Dhaka Medical College, Dhaka-1000

Bangladesh. Tel: 01670024297

E-mail: nilidr2008@yahoo.com,nilidr@gmail.com produced by Enbterobacteriaceae mainly by Escherichia coli, Klebsiella pneumoniae and oxytoca. ${ }^{2}$ They have been detected in other gram-negative bacilli such as Proteus species, Salmonella species, Pseudomonas aeruginosa and other Enteobacteriaceae. ${ }^{3-6}$ The first ESBL-producing organism was isolated in Germany in 1983. Thereafter, such organisms were reported in the USA following outbreaks of infections caused by these pathogens. ${ }^{7-9}$ The ESBL enzymes are capable of hydrolyzing broad spectrum cephalosporins and monobactams but inactive against cephamycins and imipenem. In addition, ESBL producing organisms exhibit co-resistance to many other classes of antibiotics resulting in limitation of therapeutic option. For this reason, the 
significance of such ESBL-mediated infections has been increasingly reported worldwide. ${ }^{10-13}$ The ESBL have serine at their active site and attack the amide bond in the lactam ring of antibiotics causing their hydrolysis. Because of inoculum effect and substrate specificity, their detection is a major challenge. Two indicators of ESBL are eight-fold reductions in MIC and potentiation of the inhibitor zone of third generation cephalosporin in the presence of clavulanic acid. ${ }^{14}$ For this reason, detection of ESBL, using conventional antimicrobial susceptibility methods and delay in the recognition and reporting of ESBL production by Gramnegative bacilli is associated with prolonged hospital stay, increase morbidity, motility and health care expenses. ${ }^{1}$ So, it becomes necessary to know the prevalence of these organisms and to formulate the treatment policy. This study was mainly done to determine the susceptibility of different antimicrobials to ESBL producing E. coli and K. pneumoniae isolated from various samples in Shahid Bahonar Hospital of Tehran, Iran.

The National Committee for Clinical Laboratory Standards (NCCLS) recommended that Microbiology laboratories reported ESBL-producing isolates of E. coli and Klebsiella species are resistant to all penicillins, cephalosporins (including cefepime), and aztreonam, irrespective of their individual in vitro test results. The presence of ESBL in some $K$. pneumoniae and E. coli strains poses an important challenge in clinical practice, since these organisms are common causes of serious infections. Imipenem and meropenem are considered the therapy of choice for patients with serious infections due to ESBL producing strains. Many ESBL-producing isolates are not always phenotypically resistant to oximino-cephalosporins. However, patients suffering from infections caused by ESBL-producing organisms are at risk of treatment failure if an extended spectrum of cephalosporins (ESC) are prescribed. Therefore, it is imperative for the clinical Microbiology laboratory to identify the isolates that possess increased MICs $(2 \mu \mathrm{g} / \mathrm{mL}$ ) to oximino-cephalosporins, even though they may be equal to or below the susceptibility breakpoint (MIC $8 \mu \mathrm{g} / \mathrm{mL}$ ). Retrospective study at Shahid Bahonar Hospital of Tehran demonstrated an alarming increase in the prevalence of ceftazidime-resistant $K$. pneumoniae. This led us to look into our antibiotic resistance problems that may be being caused by the ESBL with our hospitalized patients as well as those attending the outpatient clinic.

\section{Methods}

During the period July, 2007 to June, 2008, various specimens (blood, fluid, urine, swabs, tracheal aspirates/sputum) were processed for significant bacteremia in the Microbiology laboratory of Shahid Bahonar Hospital, Tehran from clinically suspected patients. This study was done on 115 Gram-negative bacilli that were confirmed as ESBL producing isolates. Medical and demographic data of the patients were collected using patients files. Data recorded were as follows: demographic (age, sex, occupation), presence of urinary catheter/ abdominal tubes/ respiratory tubes/others, admission ward etc. Samples were collected aseptically in sterilized bottles or disposable sterile tubes and submitted to clinical Microbiology laboratory. The specimens received were inoculated on blood and MacConkey agar plates. Then all plates were incubated at $37^{\circ} \mathrm{C}$ for 24 hours. Significant isolates were identified as species level using conventional bacteriological methods.

Antimicrobial susceptibility testing: Isolates were screened initially using Kirby-Bauer method and MIC assays using microdilution method, All ESBL producing isolates were confirmed using the Clinical and Laboratory Standards Institute (CLSI, formerly NCCLS 2004) ${ }^{19}$ approved doubledisk diffusion method. A positive result required an increased zone $(5 \mathrm{~mm})$, using combination disk technique with antibiotic disks containing ceftazidime $(30 \mu \mathrm{g})$, cefpodoxime $(30 \mu \mathrm{g})$ and cefotaxime $(30 \mu \mathrm{g})$ either alone or in combination with clavulanic acid $(10 \mu \mathrm{g})$. Susceptibility testing to other antibiotics was performed by disk diffusion methods as recommended by clinical laboratory standard institute (CLSI). The following antibiotic disks were used: gentamicin, amikacin, tobramycin, imipenem, meropenem, and four generation of cephalosporins (cefazoline, cephalothin, cefuroxime sodium, cefoxitin, ceftazidime, cefotaxime and cefepime), aztreonam, ampicillin, amoxicillin/clavulanate, ampicillin/sulbactam,piperacillin-tazobactam, trimethoprim/sulfamethoxazole, nitro-furantoin,ciprofloxacin, norfloxacin and nalidixic acid. According to the suggestion of CLSI, the results were interpreted. The quality control check for susceptibility testing was performed once in a week. All data were analyzed using Statistical Package for Social Sciences (SPSS).

\section{Results}

Among the patients, 68 (59.1\%) were females and 47 (40.9\%) were males. Out of 115 ESBL producing isolates, $60 \%$ were Escherichia coli and $40 \%$ were Klebsiella pneumoniae. Most of these isolates $(29.6 \%)$ were from the medical wards, followed by outpatient's clinic (24.3\%). Accident and Emergency and Surgical Units contributed 10.4\% each, followed by Intensive Care Unit (9.6\%). The least number of ESBL producing pathogens were isolated from the children wards (7\%), neonatology wards $(5.2 \%)$ and obstetric and gynecology wards $(3.5 \%)$ (Table 1$)$. 
Table-1: Rate of isolation of ESBL producing bacteria from different wards of Shahid Bahonar Hospital, Tehran.

\begin{tabular}{lcc}
\hline $\begin{array}{l}\text { Place of Sample } \\
\text { Collection }\end{array}$ & $\begin{array}{c}\text { No. of ESBL } \\
\text { producing Bacteria }\end{array}$ & Percentage (\%) \\
\hline Medical Wards & 34 & 29.6 \\
Out Patient Department & 28 & 24.3 \\
Accident and Emergency Units & 12 & 10.4 \\
Surgical Units & 12 & 10.4 \\
Intensive Care Unit & 11 & 9.6 \\
Children Wards & 8 & 7 \\
Neonatology Wards & 6 & 5.2 \\
Obstetric and Gynecology Wards & 4 & 3.5 \\
\hline
\end{tabular}

Urine $(70.4 \%)$ was the main source of ESBL producing isolates from all patients, next was the blood samples (16.5\%). Tracheal aspirates/sputum contributed to $8.7 \%$, followed by $3.5 \%$ from swabs and $0.9 \%$ from fluids (Table 2).

Table-2: Frequency and percentage of samples yielding ESBL producing isolates

\begin{tabular}{lcc}
\hline Source & Frequency & Percentage (\%) \\
\hline Urine & 81 & 70.4 \\
Blood & 19 & 16.5 \\
Tracheal aspirates/Sputum & 10 & 8.7 \\
Swabs & 04 & 3.5 \\
Fluid & 01 & 0.9 \\
Total & 115 & 100 \\
\hline
\end{tabular}

All isolates were susceptible to both imipenem and meropenem. Of all isolates, 93.9\% were susceptible to amikacin, whereas only $31.3 \%$ was susceptible to gentamicin and $20 \%$ to tobramycin. The Cephalosporins (1-4 generations) were almost $100 \%$ resistant except for Cefoxitin, which demonstrated a sensitivity of $77.4 \%$ against all isolates. For nitrofurantoin, $57.4 \%$ were sensitive. Tazocin showed a sensitivity of $49.6 \%$, whereas low sensitivity was shown towards Co-trimoxazole (13\%). Ciprofloxacin and Norfloxacin were $23.5 \%$ sensitive whereas only $17.4 \%$ were sensitive to Nalidixic acid (Table-3).
Table-3: Antimicrobial susceptibility pattern of ESBL producing $E$. coli and $K$. pneumoniae isolated from Hospital Samples

\begin{tabular}{lcc}
\hline Antibiotics & Sensitive (\%) & Resistant (\%) \\
\hline Imipenem & 100 & 0 \\
Meropenem & 100 & 0 \\
Amikacin & 93.9 & 6.1 \\
Gentamicin & 31.3 & 68.7 \\
Tobramycin & 20 & 80 \\
Cefoxitin & 77.4 & 22.6 \\
Nitrofurantoin & 57.4 & 42.6 \\
Tazocin & 49.6 & 50.4 \\
Co-trimoxazole & 13 & 87 \\
Ciprofloxacin & 23.5 & 76.5 \\
Norfloxacin & 23.5 & 76.5 \\
Nalidixic acid & 17.4 & 82.6 \\
Aztreonam & 0 & 100 \\
Ampicillin & 0 & 100 \\
Co-amoxiclav & 0 & 100 \\
Ampicillin/Sulbactam & 0 & 100 \\
\hline
\end{tabular}

High rate of resistance $(74.8 \%)$ was observed to all quinolones (ciprofloxacin, norfloxacin and nalidixic acid) tested, whereas only $5.2 \%$ of the isolates were resistant to all aminoglycosides tested. Aztreonam, ampicillin, co-amoxiclav and ampicillin/sulbactam were $100 \%$ resistant. Compared to other antibiotics both $E$. coli and $K$. pneumoniae were more sensitive to cefoxitin and amikacin. Quinolones sensitivity was greater for $K$. pneumoniae, than $E$. coli, whereas nitrofurantoin and Tazocin sensitivity was greater for E. coli. There was no difference in sensitivity to cefoxitin or amikacin, for $E$. coli or K. pneumoniae, $100 \%$ sensitivity was observed for both imipenem and meropenem. Imipenem, meropenem, cefoxitin and amikacin sensitivity were greater for blood and urine isolates. Nitrofurantoin and cefoxitin showed greater sensitivity for respiratory isolates, but quinolones were $100 \%$ resistant to respiratory and swab samples. Isolates from the neonatology wards were more sensitive compared with other wards.

\section{Discussion}

During the past decade, ESBL producing Gram-negative bacilli especially Escherichia coli and Klebsiella pneumoniae have emerged as serious pathogens both in hospital and community acquired infections worldwide. Recent studies revealed that patients with infection such as septicaemia with ESBL producing organisms had significantly higher fatality rate than those with non-ESBL isolates. ${ }^{1}$ The occurrence of ESBL among clinical isolates vary greatly world wide and geographically and are rapidly changing over time. ${ }^{15}$ Our study demonstrated clear differences in susceptibility patterns with our 115 ESBL producing isolates, between Klebsiella 
pneumoniae and Escherichia coli for Amikacin, Gentamicin, Trimethoprim-Sulfamethoxazole, Tazocin, Nitrofurantoin, Fluoroquinolones and Cefoxitin. Studies at other centres reported susceptibility patterns similar to our results for some antimicrobials; however, none of the studies have provided patterns identical to those of our study. ${ }^{16-18}$ This is probably because the ESBL is located on a plasmid that can be transferred from one organism to another rather easily and can incorporate genetic material coding for resistance to other antimicrobial classes. The high percentage of ESBL producing isolates from outpatient clinics and accident and emergency wards should alert the physician in the primary care regarding the complication of uncontrolled prescription of oral cephalosporin, such as, cefuroxime. It should also alert them regarding the probability of ESBL producing isolates in infections not responding to the first line antibiotics, such as amoxicillin. Urine $(70.4 \%)$ was the main source of ESBL producing isolates from all patients, followed by blood $(16.5 \%)$. Generally, the pathogens isolated from medical wards and from blood appeared to be more sensitive than isolates from other areas and source. Respiratory and other swab samples did not demonstrate any sensitivity for all the quinolones and trimethoprim-sulfamethiazole tested. Tobramycin demonstrated a $100 \%$ resistance towards all isolates. There are very limited treatment options available for these pathogens. So prevention remains a significant priority in controlling the development and spread of ESBL producing organisms. Several studies have demonstrated that a modifiable risk factor for the development of ESBLproducing organisms is the use of third-generation cephalosporins. Hence, formulary modification by decreasing the use of third-generation cephalosporins and increasing the use of imipenem, meropenem with amikacin or piperacillintazobactam should significantly decrease the isolation of ESBL-producing bacteria. The ESBL-producing organisms are increasing rapidly and becoming a major problem in the area of infectious diseases. In one study from Turkey the prevalence rate of ESBLs was $12-47 \%$. High rates of thirdgeneration cephalosporin use have been implicated as a major cause of this problem. Problems associated with ESBL producing isolates include multidrug resistance, difficulty in detection and treatment, and increased mortality of patients. Of all available anti-microbial agents, carbapenem are the most sensitive and reliable treatment options for infections caused by ESBL producing isolates. However, overuse of carbapenem may lead to resistance of other gram-negative organisms. Therefore, restricting the use of third-generation cephalosporins, along with implementation of infection control measures, are the most effective means of controlling and decreasing the spread of ESBL producing isolates.

\section{References}

1. Mehrgan H, Rahbar M. Prevalence of extended-spectrum beta-lactamase producing Escherichia coli in a tertiary care hospital in Tehran, Iran. Int. J. Antimicrob. Agents 2008; 31: 147-51.

2. Philippon A, Labia R, Jacoby G. Extended-spectrum lactamases. Antimicrob Agents Chemother 1989; 33: 1131-6.

3. Astal Z, Sharif FA, Abdallah SA, Fahd MI. Extended spectrum beta-lactamases in $E$. coli isolated from community-acquired urinary tract infections in the Gaza Strip, Palestine. Ann Saudi Med 2004; 24: 55-7.

4. Shah AA, Hasan F, Ahmed S, Hameed A. Characteristics, epidemiology and clinical importance of emerging strains of Gram-negative bacilli producing extended-spectrum beta lactamase. Res. Microbiol 2004; 155: 409-21.

5. Bhattacharya S. ESBL-from petri dish to the patient. Indian J.Med. Microbiol 2006; 24:20-4.

6. Vandana KE, Honnavar P. AmpC Beta Lactamase among ESBL producing $E$. coli and .If you don't look, you won't find. J. Clin. Diag. Res 2009; 3: 1635-56.

7. Quinn JP, Miyashiro D, Sahm D, Flamm R, Bush K. Novel plasmid-mediated beta-lactamase (TEM-10) conferring selective resistance to ceftazidime and aztreonam in clinical isolates of Klebsiella pneumoniae. Antimicrob Agents Chemother 1989; 33: 1451-1456.

8. Knothe A, Shah P, Kremery V, Antal M, Mitsuhashi S. Transferable resistance to cefotaxime, cefoxitin, cefamandole and cefuroxime and in clinical isolates of Klebsiella pneumoniae and Serratia marcescens. Infection 1983; 11: 315-7.

9. Jacoby GA, Medeiros AA, O'Brien TF, Pinto ME, Jiang $\mathrm{J}$. Broad-spectrum transmissible -lactamases. $\mathrm{N}$ Engl J Med 1989; 319: 723-4.

10. Winokur PL, Canton R, Casellas JM, Legakis N. Variations in the prevalence of strains expressing an extended spectrum beta-lactamase phenotype and characterisation of isolates from Europe, the Americas and the Western Pacific region. Clin Infect Dis 2001; 32 (suppl 2): S94-S103.

11. Bradford PA. Extended-spectrum beta-lactamases in the $21^{\text {st }}$ century: characterisation, epidemiology, and detection of this important resistance threat. Clin Microbiol Rev 2001; 14: 933-951.

12. Khanfar HS, Bindayna KM, Senok AC, Botta GA. Extended spectrum beta-lactamases (ESBL) in E. coli and $K$. pneumoniae: trends in the hospital and community settings. J. Infect. Dev. Countries 2009; 3: 295-9.

13. Aminzadeh Z, Sadat KM, Sha'bani M. Bacteriuria by 
extended spectrum Beta-lactamase-producing E. coli and Klebsiella pneumoniae: isolates in a governmental hospital in South of Tehran, Iran. Iran J. Kidney Dis 2008; 2: 197-200.

14. Chaudhary U, Aggarwal R. Extended spectrum betalactamases (ESBL) - An emerging threat to clinical therapeutics. Indian J Med Microbiol 2004;22:75-80.

15. Babypadmini S, Appalaraju B. Extended spectrum lactamase in urinary isolates of E. coli and Klebsiella pneumoniae - prevalence and susceptibility pattern in a Tertiary care hospital. Indian J. Med. Microbiol 2004; 22: $172-4$.

16. Paterson DL, Ko WC, Von Gottberg A, Casellas JM, Mulazimoglu L, Klugman KP et al. Outcome of cephalosporin treatment for serious infections due to apparently susceptible organisms producing extendedspectrum beta-lactamases: implications for the clinical microbiology laboratory. J Clin Microbiol 2001; 39: 2206-12.

17. Thomson KS, Moland ES. Cefepime, piperacillintazobactam, and the inoculum effect in tests with extended-spectrum beta-lactamase producing Enterobacteriaceae. Antimicrob Agents Chemother 2001; 45: 3548-54.

18. Quale JM, Landman D, Bradford PA, Visalli M, Ravishankar J, Flores C, et al. Molecular epidemiology of a citywide outbreak of extended-spectrum beta-lactamase producing Klebsiella pneumoniae infection. Clin Infect Dis 2002; 35: 834-41.

19. National Committee for Clinical Laboratory Standards. NCCLS. NCCLS document M 100-S15. Performance Standards for Antimicrobial Susceptibility Testing. 8th ed. Pennsylvania: NCCLS: Wayne; 2004. 